\title{
Impact of surgical pulmonary valve replacement on ventricular strain and synchrony in patients with repaired tetralogy of Fallot: a cardiovascular magnetic resonance feature tracking study
}

Sowmya Balasubramanian ${ }^{1,2^{*}}$ (D) David M. Harrild ${ }^{1,2}$, Basavaraj Kerur ${ }^{1}$, Edward Marcus ${ }^{1,2}$, Pedro del Nido ${ }^{3,4}$, Tal Geva ${ }^{1,2}$ and Andrew J. Powell ${ }^{1,2}$

\begin{abstract}
Background: In patients with repaired tetralogy of Fallot (TOF), a better understanding of the impact of surgical pulmonary valve replacement (PVR) on ventricular mechanics may lead to improved indications and outcomes. Therefore, we used cardiovascular magnetic resonance (CMR) feature tracking analysis to quantify ventricular strain and synchrony in repaired TOF patients before and after PVR.

Methods: Thirty-six repaired TOF patients (median age 22.4 years) prospectively underwent CMR a mean of $4.5 \pm 3$. 8 months before PVR surgery and $7.3 \pm 2.1$ months after PVR surgery. Feature tracking analysis on cine steady-state free precession images was used to measure right ventricular (RV) and left ventricular (LV) circumferential strain from short-axis views at basal, mid-ventricular, and apical levels; and longitudinal strain from 4-chamber views. Intraventricular synchrony was quantified using the maximum difference in time-to-peak strain, the standard deviation of the time-to-peak, and cross correlation delay (CCD) metrics; interventricular synchrony was assessed using the CCD metric.

Results: Following PVR, RV end-diastolic volume, end-systolic volume, and ejection fraction declined, and LV end-diastolic volume and end-systolic volume both increased with no significant change in the LV ejection fraction. LV global basal and apical circumferential strains, and basal synchrony improved. RV global circumferential and longitudinal strains were unchanged, and there was a varied impact on synchrony across the locations. Interventricular synchrony worsened at the midventricular level but was unchanged at the base and apex, and on 4-chamber views.
\end{abstract}

Conclusions: Surgical PVR in repaired TOF patients led to improved LV global strain and no change in RV global strain. LV and RV synchrony parameters improved or were unchanged, and interventricular synchrony worsened at the midventricular level.

Keywords: Tetralogy of fallot, Pulmonary valve replacement, Feature tracking, Myocardial strain, Ventricular synchrony

\footnotetext{
* Correspondence: sowmyab@med.umich.edu

Dr. Mark Fogel served as a Guest Editor for this manuscript.

${ }^{1}$ Department of Cardiology, Boston Children's Hospital, Boston, USA

2Department of Pediatrics, Harvard Medical School, Boston, USA

Full list of author information is available at the end of the article
}

(c) The Author(s). 2018 Open Access This article is distributed under the terms of the Creative Commons Attribution 4.0 International License (http://creativecommons.org/licenses/by/4.0/), which permits unrestricted use, distribution, and reproduction in any medium, provided you give appropriate credit to the original author(s) and the source, provide a link to the Creative Commons license, and indicate if changes were made. The Creative Commons Public Domain Dedication waiver (http://creativecommons.org/publicdomain/zero/1.0/) applies to the data made available in this article, unless otherwise stated. 


\section{Background}

Young children undergoing surgical repair of tetralogy of Fallot (TOF) have excellent short-term survival [1]; however, they experience significant morbidity and mortality related to biventricular dysfunction and arrhythmia in their adult years [2-4]. These sequelae are believed to be in part related to chronic pulmonary regurgitation (PR) caused by efforts to relieve pulmonary valve stenosis with the initial repair. Thus, surgical pulmonary valve replacement (PVR) is often performed subsequently to improve the long-term outcome $[5,6]$. Although a reduction in PR, right ventricular (RV) end-diastolic volume (EDV), and RV end-systolic volume (ESV) is observed, the beneficial impact of PVR on ventricular systolic function, exercise capacity, arrhythmia, and survival is uncertain [6-9].

A better understanding of the effects of surgical PVR on ventricular function may lead to improved indications and outcomes for the procedure. In particular, areas that merit further investigation are regional ventricular mechanics and synchrony. In repaired TOF patients, right bundle branch block is nearly universal and several studies have documented dyssynchronous ventricular contraction [10-12]. Moreover, left ventricular (LV) dysfunction is seen in conjunction with RV dysfunction suggesting adverse ventricular-ventricular interactions [3, 13, 14]. Nevertheless, our knowledge of regional ventricular mechanics in this patient group remains rudimentary, in part because robust techniques for its assessment have not been applied.

Feature tracking is an image processing technology that quantifies myocardial tissue deformation, and has been increasingly employed to aid cardiac resynchronization therapy $[15,16]$. This technique has primarily been applied to ultrasound images; however, recent work has adapted and validated its use with cardiovascular magnetic resonance (CMR) images in both the right and left ventricles [12, 17-21]. CMR offers the advantage over echocardiography of consistent high-quality imaging of both ventricles in patients with repaired TOF.

Our group previously published a prospective, randomized study comparing 2 techniques for surgical PVR: PVR alone versus PVR plus RV remodeling with the resection of scar tissue [22]. No significant difference was observed in the primary outcome-change in $\mathrm{RV}$ ejection fraction (EF) measured by $\mathrm{CMR}-$ or in any of the secondary outcomes at 6-month postoperative follow-up. In this study, we performed feature tracking image analysis on data from the trial cohort to determine the impact of surgical PVR on strain and ventricular synchrony in patients with repaired TOF.

\section{Methods}

\section{Subjects}

Subjects in this study were all participants in a previously reported prospective, randomized, single-center trial comparing 2 techniques for surgical PVR [22]. These patients were $>10$ years of age with repaired TOF or similar physiology presenting for PVR between February 2004 and October 2008. Other inclusion criteria in the prior study included chronic PR (regurgitation fraction by CMR $\geq 25 \%$ ) and at least 2 of the following conditions: RV EDV index $\geq 160 \mathrm{ml} / \mathrm{m}^{2}$, RV ESV index $\geq 70 \mathrm{ml} / \mathrm{m}^{2}$, RV EF $\leq 45 \%$, LV EDV index $\leq 65 \mathrm{ml} / \mathrm{m}^{2}$, RV outflow aneurysm, or a clinical criterion such as exercise intolerance, symptoms and signs of heart failure, or cardiac medications. Exclusion criteria included any of the following: severe RV outflow obstruction, severe RV hypertension with RV pressure $\geq$ systemic pressure, and contraindications to preoperative CMR. Patients were randomized to PVR alone or a combination of PVR with RV remodeling surgery. The study protocol included a pre-operative and a 6-month post-operative CMR study.

For the current study, we analyzed the database of the prospective trial, selected only those patients with TOF, and performed feature tracking analysis of the CMR images. The Boston Children's Hospital Committee on Clinical Investigation granted permission for this study and waived the requirement for informed consent.

\section{CMR}

The CMR protocol used for patients with repaired TOF has been previously published [23]. Briefly, studies were performed with a 1.5-T whole body scanner (Achieva, Phillips Healthcare Systems, Best, the Netherlands or TwinSpeed, GE Healthcare, Milwaukee, Wisconsin, USA) using surface coils selected based on patient size. The scanner manufacturer was different for the pre- and post-PVR CMR study in 4 patients. Imaging included breath-hold, electrocardiographically-gated, balanced steady-state free precession cine CMR acquisitions in 4-chamber and short-axis planes. A total of 12-14 slices were obtained in the short-axis plane to completely cover both ventricles. In all views, 30 images per cardiac cycle were acquired which yielded a temporal resolution of 20-40 ms, depending on heart rate. Ventricular volumes and blood flow were measured using commercially available software (QMASS and QFLOW, Medis, Leiden, the Netherlands) [23].

\section{Strain analysis}

Feature tracking analysis for determination of strain was performed using commercially available software (Diogenes v. 1.1.02, TomTec Imaging Systems, Unterschleissheim, Germany), as previously described [12]. Circumferential 
strains for the LV and RV were measured from short-axis views at 3 levels: basal, mid-ventricular, and apical. The mid-ventricular level was first identified, and the basal and apical slices were specified to be equidistant from the mid-ventricular slice. The LV outflow tract was not included in the basal slice. Pre- and post-PVR images on each subject were compared to ensure similar slice locations. For short-axis views, the myocardium of each ventricle was divided equally into 6 segments at the mid and basal locations, and 4 segments at the apical location (Fig. 1a). Longitudinal strains for the LV and RV were measured from the 4-chamber view with division into 6 segments (Fig. 1b). Global peak strain for each view was calculated as the average of the peak strains for each segmental curve $[17,24]$. Strain measurements were performed by the same investigator in all patients in order to promote consistency. In addition, they were repeated by that investigator and performed by a second investigator in a random sample of patients to assess intraobserver and interobserver agreement. Note that strain is calculated as follows: (final length - initial length) / initial length. Thus, shortening in the longitudinal and circumferential directions yields a negative strain number, and that the more negative the number, the better the shortening.

\section{Synchrony analysis}

Various ventricular synchrony parameters based on feature tracking measurements have been reported; however, there is no consensus on the optimal approach. Hence, we used 3 techniques that have been reported in the literature as measures of ventricular synchrony $[17,25,26]$. Three ventricular synchrony parameters were calculated for each ventricle on each of the 3 short-axis views and the 4-chamber view based on the

\section{a}
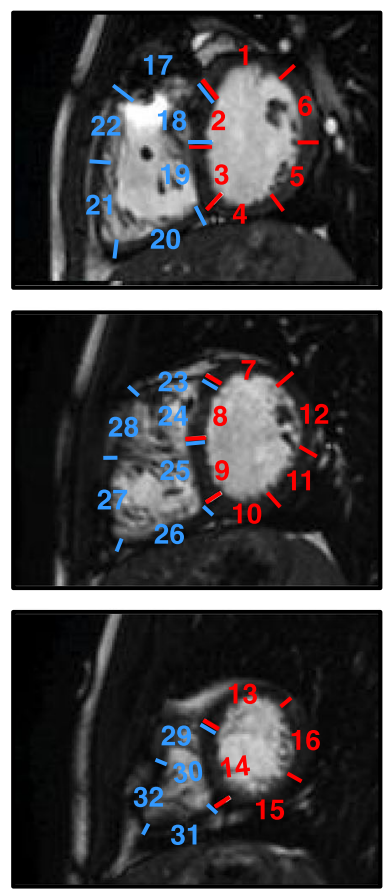

b

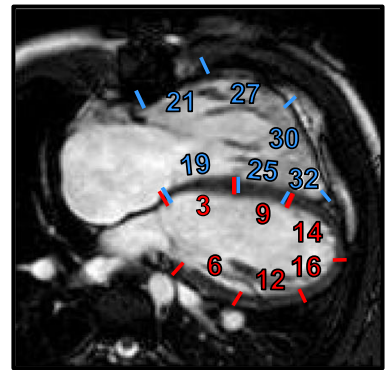

Basal Right Ventricle

17 Superior

18 Anteroseptal

19 Inferoseptal

20 Inferior

21 Anterior

22 Anterosuperior

Mid Right Ventricle

23 Superior

24 Anteroseptal

25 Inferoseptal

26 Inferior

27 Anterior

28 Anterosuperior

Apical Right Ventricle

29 Superior

30 Septal

31 Inferior

32 Anterior
Basal Left Ventricle

1 Anterior

2 Anteroseptal

3 Inferoseptal

4 Inferior

5 Inferolateral

6 Anterolateral

Mid Left Ventricle

7 Anterior

8 Anteroseptal

9 Inferoseptal

10 Inferior

11 Inferolateral

12 Anterolateral

\section{Apical Left Ventricle}

13 Anterior

14 Septal

15 Inferior

16 Lateral

\begin{tabular}{llll} 
Right Ventricle & \multicolumn{2}{l}{ Left Ventricle } \\
19 & Basal inferoseptal & 3 & Basal inferoseptal \\
21 & Basal anterior & 6 & Basal anterolateral \\
25 & Mid inferoseptal & 9 & Mid inferoseptal \\
27 & Mid anterior & 12 & Mid anterolateral \\
30 & Apical septal & 14 & Apical septal \\
32 & Apical anterior & 16 & Apical lateral
\end{tabular}

Right Ventricle Left Ventricle

3 Basal inferoseptal

Basal anterolatera

12 Mid anterolateral

14 Apical septal

Apical lateral

\footnotetext{
Fig. 1 Schematic diagram illustrating the division of the right ventricle (RV) and left ventricle (LV) myocardium into segments on short-axis (a) and 4chamber views (b)
} 
strain versus time data: 1) the maximum difference in time-to-peak strain among any 2 of the segments (latest versus earliest segment), 2) the standard deviation of the time-to-peak strain values for all segments, and 3) the cross-correlation delay (CCD). The time to peak strain was measured from the QRS trigger to the peak strain time. Using a custom built virtual instrument (LabVIEW 8.2, National Instruments, Austin, Texas, USA), the CCD was measured by iteratively shifting 1 curve in time relative to a second curve in a stepwise fashion, and calculating the normalized correlation coefficient between the curves for each time shift [25, 27]. The time shift that resulted in the maximum correlation coefficient was defined as the CCD between the 2 curves. Rather than relying on just the peak points, CCD takes into account the entire strain versus time curve to determine the temporal offset. For each ventricle on each of the 4 views, the CCDs were measured between opposing segments, and the largest value of the opposing segment CCDs was reported. In addition, the interventricular CCD on each of the 4 views was calculated by comparing the global strain versus time curves for the LV and RV.

\section{Statistical analysis}

All collected data were tested for normalcy using the Shapiro-Wilk test. Continuous data are presented as median (range) and mean \pm standard deviation. A two-tailed paired t-test was used to compare pre- and post-PVR strain and synchrony parameters. A $p$-value $\leq 0.05$ was considered statistically significant. The intraclass correlation coefficient (ICC) and mean difference were used to assess intraobserver and interobserver agreement.

\section{Results}

\section{Patients}

Of the 64 patients in the initial prospective trial, 36 had a diagnosis of TOF as well as pre- and post-operative CMR studies with suitable image quality for strain analysis. These patients constitute the study group, and their demographic and clinical data are summarized in Table 1. Thirteen patients were randomized to the PVR alone group and 23 to the PVR with RV remodeling surgery group. CMR examinations occurred at a mean of $4.5 \pm 3.8$ (range 0.03-12.7) months before PVR surgery and $7.3 \pm 2.1$ (range 5.2-10.8) months after surgery.

\section{Diagnostic testing results}

Key diagnostic testing results pre- and post-PVR are shown in Table 2. As expected, PVR led to virtual elimination of $\mathrm{PR}$, and a significant decline in RV EDV and ESV. These changes yielded a slight decline in RV EF. The LV EDV and ESV both increased slightly with no
Table 1 Patient characteristics $(n=36)$

\begin{tabular}{ll}
\hline Male & $23(64 \%)$ \\
Prior aortopulmonary shunt & $10(28 \%)$ \\
Age at initial complete repair (years) & $1.5(0.0-19.4)$ \\
Age at PVR (years) & $22.4(12.3-57.7)$ \\
Cardiac symptoms prior to PVR & $21(58 \%)$ \\
NYHA Class I or II prior to PVR & $33(92 \%)$ \\
NYHA Class III or IV prior to PVR & $3(8 \%)$ \\
Prescribed cardiac medications prior to PVR & $16(44 \%)$ \\
Additional procedures with PVR & \\
Closure of patent foramen ovale or atrial & $8(22 \%)$ \\
septal defect & \\
Pulmonary artery plasty & $10(28 \%)$ \\
Tricuspid annuloplasty & $6(17 \%)$ \\
Cryoablation & $2(6 \%)$ \\
Maze procedure & $4(11 \%)$ \\
Closure of ventricular septal defect & $3(8 \%)$ \\
Other & $4(11 \%)$ \\
\hline
\end{tabular}

Data presented as $\mathrm{n}$ (\%) or median (range)

PVR pulmonary valve replacement

significant change in the LV EF. There was no significant change in QRS duration or the heart rate at the time of CMR.

\section{Strain analysis}

Global strain values pre- and post-PVR are shown in Fig. 2. In the LV, global circumferential strain at the base and apex improved following PVR; strain at the mid ventricle also tended to improve but this did not reach statistical significance. Global longitudinal strain was unchanged. In the RV, global circumferential strain at all 3 levels and global longitudinal strain were unchanged.

The impact of PVR on segmental strain in the LV and RV is shown in Fig. 3. Overall, changes in regional stain were minimal. Significant differences in circumferential LV strain were seen in 2 of 16 segments, and these were both improvements. Longitudinal strain changed in 1 of 6 segments and this was a decline. Significant differences in circumferential RV strain were seen in 2 of 16 segments, 1 increase and 1 decrease. Longitudinal strain changed in 1 of 6 segments and this was a decline.

\section{Synchrony analysis}

Synchrony parameters derived from the segmental strain versus time curves pre- and post-PVR are shown in Table 3. For LV circumferential strain, all 3 parameters showed significantly improved synchrony post-PVR at the base. At the mid and apex levels, all parameters tended to improve and some met the significance threshold. There were no significant synchrony changes based on longitudinal strain. 
Table 2 Diagnostic testing results pre- and post-PVR $(n=36)$

\begin{tabular}{|c|c|c|c|}
\hline Parameter & $\begin{array}{l}\text { Pre- } \\
\text { PVR }\end{array}$ & $\begin{array}{l}\text { Post- } \\
\text { PVR }\end{array}$ & $P$-value \\
\hline \multicolumn{4}{|l|}{ Electrocardiogram } \\
\hline QRS duration (ms) & $153 \pm 38$ & $152 \pm 39$ & 0.99 \\
\hline \multicolumn{4}{|l|}{ Exercise Testing } \\
\hline Peak $\mathrm{VO}_{2}(\mathrm{ml} / \mathrm{kg} / \mathrm{min})$ & $26 \pm 9$ & $25 \pm 8$ & 0.31 \\
\hline \multicolumn{4}{|l|}{ Echocardiography } \\
\hline$\leq$ Mild tricuspid regurgitation & 33 & 35 & \\
\hline$\geq$ Moderate tricuspid regurgitation & 3 & 1 & \\
\hline $\begin{array}{l}\text { RV systolic pressure by tricuspid } \\
\text { regurgitation jet velocity }\end{array}$ & $35 \pm 15$ & $25 \pm 7$ & 0.009 \\
\hline \multicolumn{4}{|l|}{ CMR } \\
\hline Heart rate & $75 \pm 11$ & $73 \pm 13$ & 0.38 \\
\hline $\operatorname{LVEDV}_{i}\left(\mathrm{ml} / \mathrm{m}^{2}\right)$ & $88 \pm 17$ & $93 \pm 17$ & 0.01 \\
\hline LV ESV $V_{i}\left(\mathrm{ml} / \mathrm{m}^{2}\right)$ & $37 \pm 10$ & $40 \pm 12$ & 0.02 \\
\hline LV ejection fraction (\%) & $58 \pm 8$ & $57 \pm 7$ & 0.35 \\
\hline $\operatorname{RV} \operatorname{EDV}_{\mathrm{i}}\left(\mathrm{ml} / \mathrm{m}^{2}\right)$ & $194 \pm 34$ & $119 \pm 16$ & $<0.001$ \\
\hline $\operatorname{RV} \operatorname{ESV}_{\mathrm{i}}\left(\mathrm{ml} / \mathrm{m}^{2}\right)$ & $100 \pm 24$ & $65 \pm 16$ & $<0.001$ \\
\hline RV ejection fraction (\%) & $49 \pm 7$ & $46 \pm 7$ & 0.02 \\
\hline Pulmonary regurgitation fraction (\%) & $48 \pm 10$ & $4 \pm 5$ & $<0.0001$ \\
\hline
\end{tabular}

For the RV, both of the time-to-peak parameters showed significantly improved synchrony at the base and worsened at the apex and longitudinally. At the mid location, there were no significant synchrony changes.

In addition to the synchrony parameters reported above, the raw time-to-peak strain values (rather than differences) for each segment were compared pre- and post-PVR. This analysis provides information on how PVR affects the timing of contraction relative to the cardiac cycle rather than assessing synchrony per se. All LV segments with a statistically significant change in time-to-peak strain following PVR were increases and these were 2 of the 6 longitudinal and 7 of 16 circumferential segments (Fig. 4a). Similarly, all RV segments with a statistically significant change in time-to-peak strain after PVR were increases, and these were 1 of the 6 longitudinal and 4 of the 16 circumferential segments (Fig. 4b).

Interventricular synchrony was assessed by comparing the global strain versus time curves for the LV and RV on each of the 4 views, and calculating the CCDs. The LV peak strain preceded RV peak strain in 128 of 144 segments (89\%) pre-PVR and in 127 of the 144 segments $(88 \%)$ post PVR. The interventricular CCD significantly increased after PVR at the mid-ventricular level $(50.4 \pm 26.4 \mathrm{~ms}$ vs. $66.7 \pm 29.1 \mathrm{~ms}, p=0.002)$, as a result of slightly earlier LV peak strain. There was no change in the interventricular CCD at the base (76.5 \pm

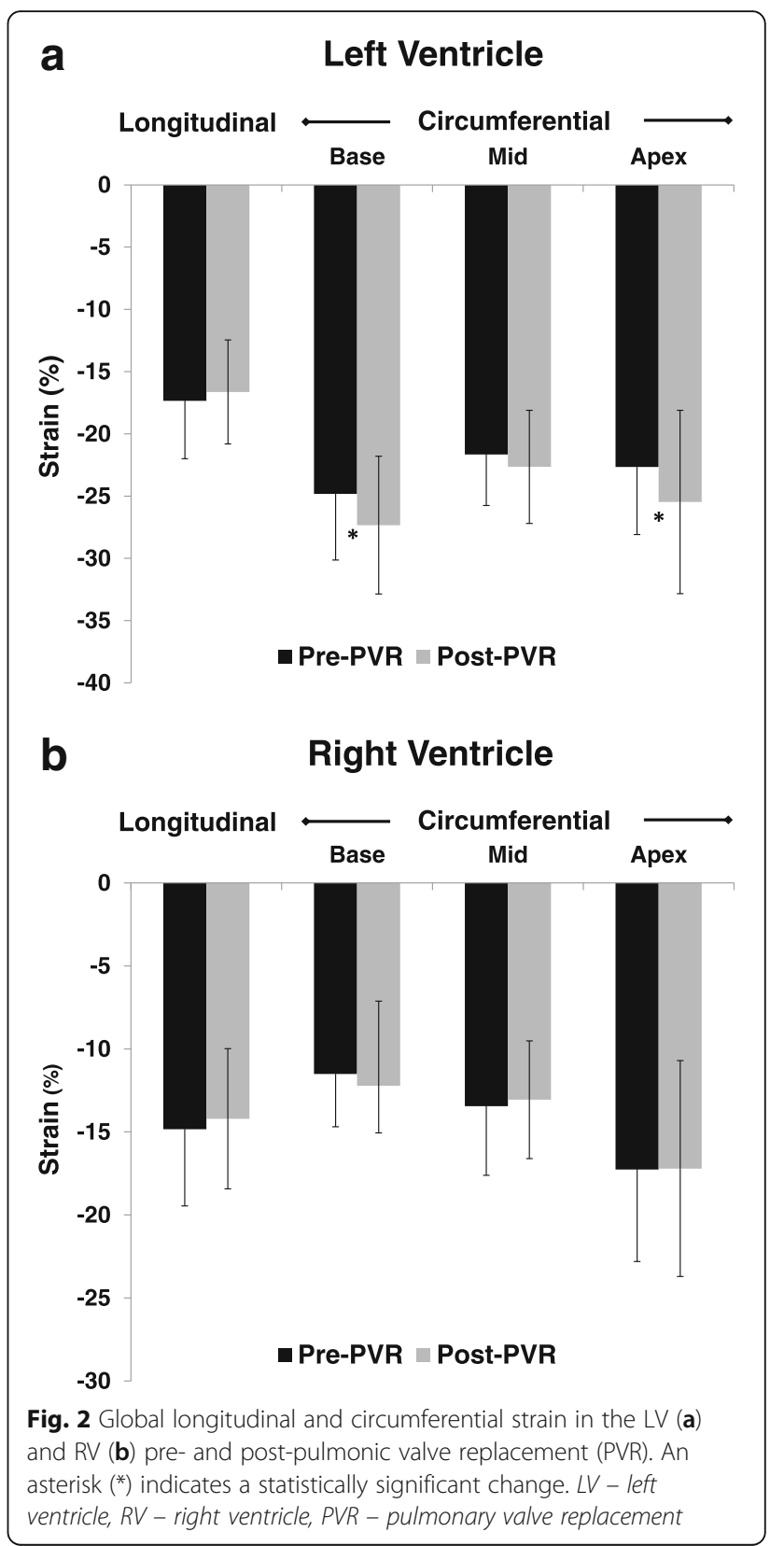

$53.8 \mathrm{~ms}$ vs. $90.5 \pm 56.2 \mathrm{~ms}, p=0.62)$, the apex $(46.8 \pm$ $34.4 \mathrm{~ms}$ vs. $49.0 \pm 33.2 \mathrm{~ms}, p=0.73)$, or the 4 -chamber view $(61.8 \pm 109.6 \mathrm{~ms}$ vs. $52.3 \pm 86.7 \mathrm{~ms}, p=0.69)$.

\section{Intraobserver and interobserver agreement}

Table 4 shows the intraobserver and interobserver agreement results for global strain and synchrony parameters. Intraobserver agreement was good for LV circumferential and longitudinal strain, and for RV circumferential strain (all ICCs $\geq 0.93$ ), and acceptable for RV longitudinal strain (ICC 0.85). Interobserver agreement for these parameters was lower. Compared to global strains, time-to-peak strain and CCD generally had lower ICCs; 


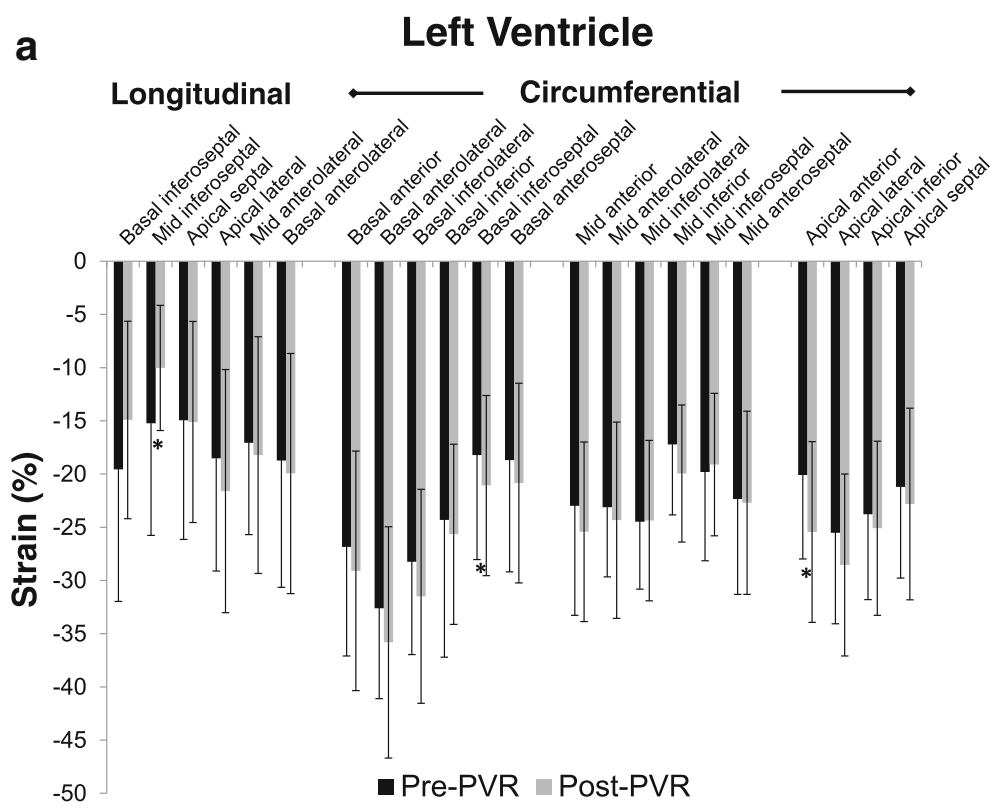

b

Right Ventricle

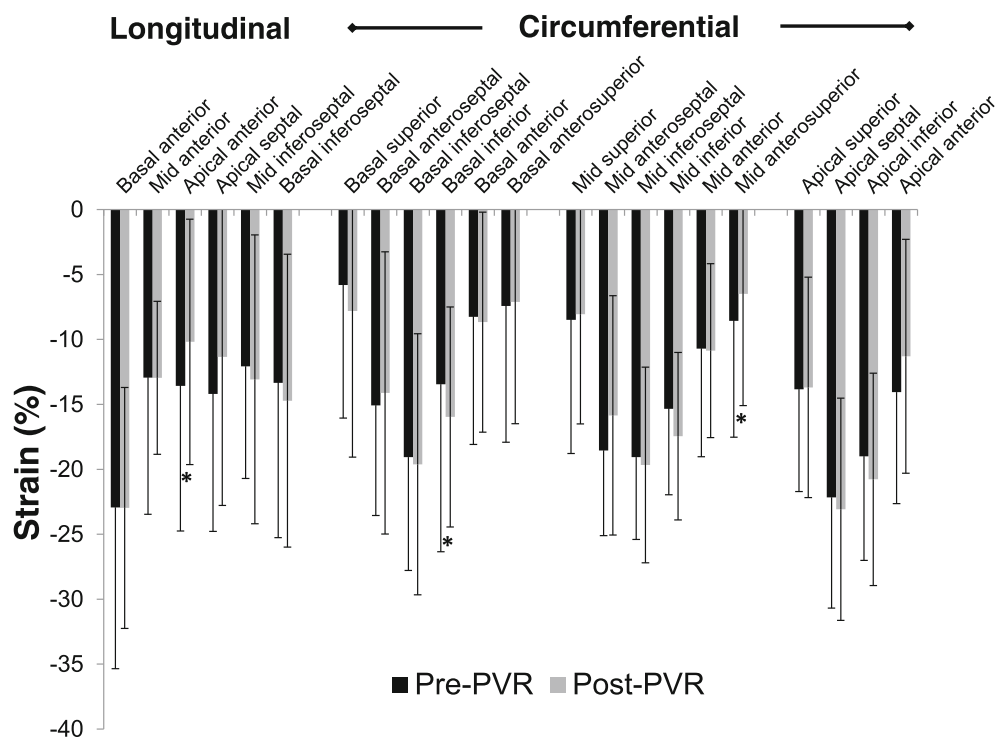

Fig. 3 Segmental longitudinal and circumferential strain in the LV (a) and RV (b) pre- and post-PVR. An asterisk (*) indicates a statistically significant change. $L V$ - left ventricle, $R V$ - right ventricle, PVR - pulmonary valve replacement

nevertheless, intraobserver ICCs for these LV synchrony parameters were all $\geq 0.73$. Agreement for RV synchrony values derived from longitudinal strain curves was only fair.

\section{Discussion}

This is the first study to use CMR to assess the impact of surgical PVR on myocardial strain and synchrony in patients with repaired TOF. At a mean of 7 months following PVR, LV global basal and apical strain, and basal synchrony improved. PVR had no impact on RV global circumferential and longitudinal strains, and a varied impact on synchrony across the locations. Interventricular synchrony worsened at the midventricular level but was unchanged at the base and apex, and on 4-chamber views. These results provide new insight into the effects of PVR on ventricular mechanics and may prompt modifications in management.

Based on multiple studies published over the past decade in repaired TOF patients, a clear picture has 
Table 3 Synchrony parameters pre- and post-PVR $(n=36)$

\begin{tabular}{|c|c|c|c|}
\hline Parameter & Pre-PVR & Post-PVR & $P$-value \\
\hline \multicolumn{4}{|l|}{ Left ventricle } \\
\hline \multicolumn{4}{|l|}{ Longitudinal strain } \\
\hline Maximum difference in time-to-peak & $315 \pm 185$ & $351 \pm 196$ & 0.33 \\
\hline Standard deviation of time-to-peak & $121 \pm 73$ & $139 \pm 82$ & 0.23 \\
\hline Cross-correlation delay & $301 \pm 218$ & $340 \pm 242$ & 0.39 \\
\hline \multicolumn{4}{|l|}{ Circumferential strain: base } \\
\hline Maximum difference in time-to-peak & $233 \pm 172$ & $151 \pm 120$ & 0.01 \\
\hline Standard deviation of time-to-peak & $89 \pm 63$ & $58 \pm 48$ & 0.02 \\
\hline Cross-correlation delay & $217 \pm 182$ & $107 \pm 77$ & 0.0001 \\
\hline \multicolumn{4}{|l|}{ Circumferential strain: mid } \\
\hline Maximum difference in time-to-peak & $124 \pm 75$ & $107 \pm 55$ & 0.02 \\
\hline Standard deviation of time-to-peak & $49 \pm 27$ & $43 \pm 20$ & 0.15 \\
\hline Cross-correlation delay & $106 \pm 113$ & $77 \pm 53$ & 0.13 \\
\hline \multicolumn{4}{|l|}{ Circumferential strain: apex } \\
\hline Maximum difference in time-to-peak & $58 \pm 46$ & $47 \pm 44$ & 0.27 \\
\hline Standard deviation of time-to-peak & $28 \pm 22$ & $23 \pm 21$ & 0.26 \\
\hline Cross-correlation delay & $99 \pm 146$ & $42 \pm 24$ & 0.04 \\
\hline \multicolumn{4}{|l|}{ Right ventricle } \\
\hline \multicolumn{4}{|l|}{ Longitudinal strain } \\
\hline Maximum difference in time-to-peak & $249 \pm 176$ & $337 \pm 170$ & 0.02 \\
\hline Standard deviation of time-to-peak & $97 \pm 70$ & $128 \pm 64$ & 0.02 \\
\hline Cross-correlation delay & $258 \pm 171$ & $254 \pm 179$ & 0.91 \\
\hline \multicolumn{4}{|l|}{ Circumferential strain: base } \\
\hline Maximum difference in time-to-peak & $361 \pm 198$ & $265 \pm 155$ & 0.01 \\
\hline Standard deviation of time-to-peak & $136 \pm 70$ & $99 \pm 59$ & 0.004 \\
\hline Cross-correlation delay & $342 \pm 285$ & $341 \pm 286$ & 0.56 \\
\hline \multicolumn{4}{|l|}{ Circumferential strain: mid } \\
\hline Maximum difference in time-to-peak & $214 \pm 133$ & $268 \pm 179$ & 0.11 \\
\hline Standard deviation of time-to-peak & $96 \pm 55$ & $101 \pm 66$ & 0.73 \\
\hline Cross-correlation delay & $268 \pm 216$ & $263 \pm 224$ & 0.93 \\
\hline \multicolumn{4}{|l|}{ Circumferential strain: apex } \\
\hline Maximum difference in time-to-peak & $95 \pm 84$ & $129 \pm 97$ & 0.047 \\
\hline Standard deviation of time-to-peak & $43 \pm 37$ & $60 \pm 43$ & 0.03 \\
\hline Cross-correlation delay & $119 \pm 148$ & $126 \pm 145$ & 0.82 \\
\hline
\end{tabular}

Values expressed as mean \pm standard deviation in $\mathrm{ms}$

emerged regarding the short-term effects of surgical PVR on ventricular volumes and $\operatorname{EF}[5,6,28]$. On average, RV EDV decreases by $30-40 \%$, LV EDV increases slightly, and RV and LV EF largely remain unchanged. This response was also seen in our study cohort.

Analysis of these global parameters, however, is likely insufficient to fully characterize the effects of PVR on ventricular mechanics. Myocardial deformation indices are thought to be more sensitive markers of ventricular function than volumetric parameters [12, 13, 29]. Our finding of improved LV circumferential strain and synchrony following PVR in the absence of appreciable changes in LV EF follows this premise. The utility of echocardiography-based strain measurement techniques in repaired TOF patients is limited by diminished acoustic windows and visualization, particularly for the RV. CMR has the advantage of providing consistently high image quality for both the RV and LV myocardial walls. There are several CMR methods for myocardial deformation imaging; however, only feature tracking can be applied to standard cine images without performing additional time-consuming imaging sequences. The use of this approach for our study is further supported by reports from our group and others that have shown acceptable correlation between feature tracking and the more established myocardial tagging technique for strain measurement $[18,30,31]$. Investigations have also found that intraobserver and interobserver agreement for radial strain by CMR feature tracking is lower than that for circumferential and longitudinal strain [20, 30]. For this reason, radial strain assessment was not included in the current study. In our study, intraobserver agreement for LV and RV strain and LV synchrony parameters was good; for RV synchrony parameters, it was only fair. Interobserver agreement for several of the strain and synchrony parameters was rather low, which suggests a limited clinical role of this technique for assessing RV synchrony in particular. However, the high intraobserver reliability for most parameters is of primary importance in this study as all of the pre- and post-PVR measurements were made by a single observer.

As the optimal technique to assess ventricular synchrony is unknown, we examined the effect of PVR on 3 metrics extracted from segmental strain versus time curves-maximum difference in time-to-peak, standard deviation of time-to-peak, and CCD. In our analysis, the most robust change with PVR was a significant improvement in intraventricular RV and LV synchrony at the basal level. Interventricular synchrony did not change in 3 of the 4 views; the one statistically significant difference was a mild worsening of synchrony in the mid-ventricular view related to earlier LV contraction. QRS duration was unchanged.

Our data demonstrated for the most part concordant changes in strain and synchrony parameters in the LV supporting a causal relationship. Specifically, following PVR, most of the circumferential strain-based synchrony parameters significantly improved at the base and apex of the LV. This enhanced coordination of contraction may have contributed to the improvements in LV global circumferential strain that were also seen in the same segments. More favorable 

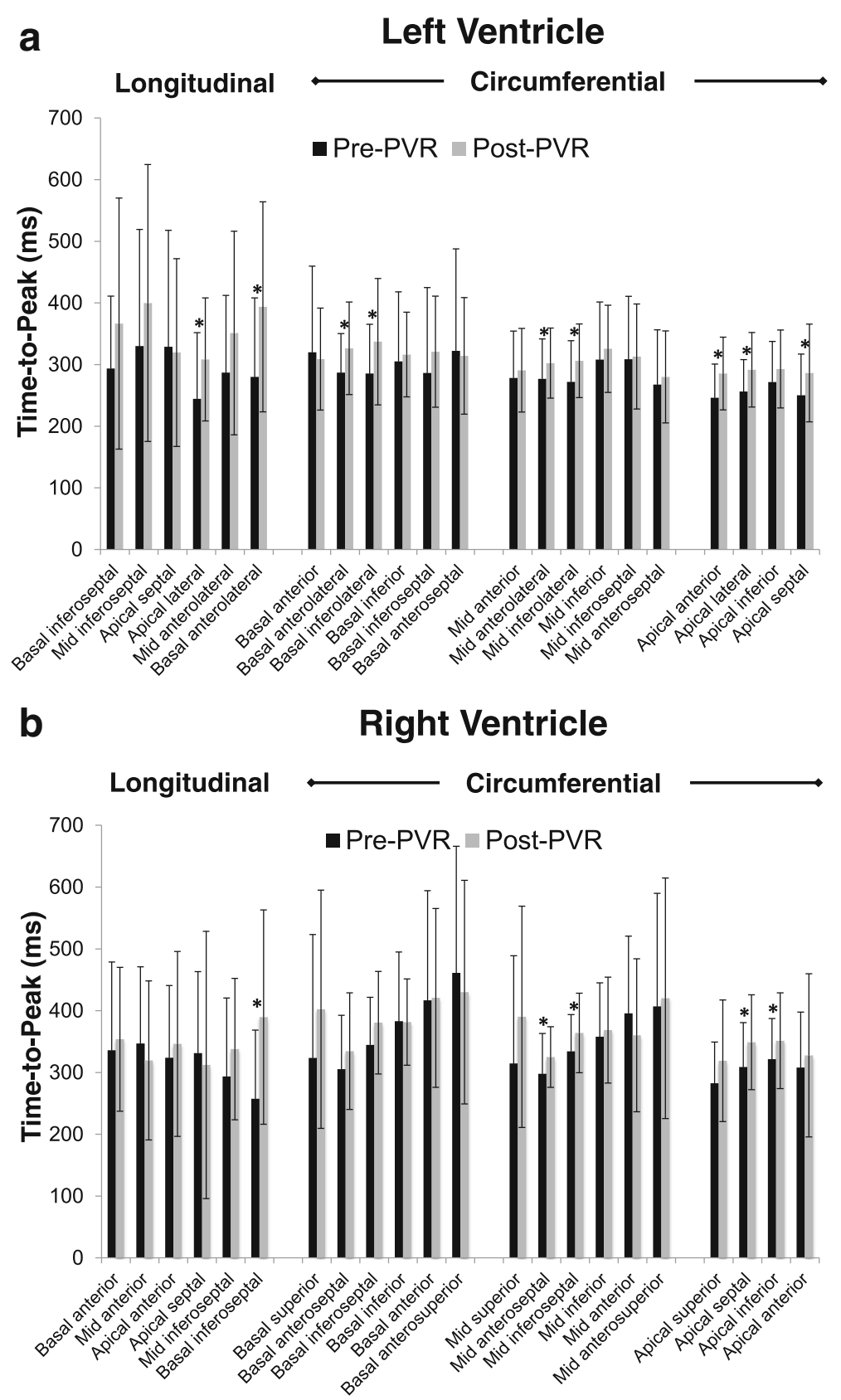

Fig. 4 Segmental time-to-peak longitudinal and circumferential strain in the LV (a) and RV (b) pre- and post-PVR. An asterisk $(*)$ indicates a statistically significant change. $L V$ - left ventricle, $R V$ - right ventricle, PVR - pulmonary valve replacement

ventricular-ventricular interactions in diastole may also have contributed to the improved LV strain [32]. The near elimination of PR with PVR would be expected to reduce the pressure difference across the ventricular septum, and, therefore, diminish diastolic septal bowing toward the LV. The resultant improved LV filling increases preload and muscle fiber stretch leading to increased strain by the Frank-Starling mechanism. This explanation is supported by the increase in indexed LV EDV seen after PVR.

\section{Prior studies}

Only a few other reports have measured changes in strain that accompany PVR. A similar study from our group examined the impact of transcatheter pulmonary valve implantation on strain assessed by CMR feature tracking [17]. All patients $(n=31)$ had RV-to-pulmonary artery conduit dysfunction, and were divided into those with predominant pulmonary stenosis $(n=18)$ and those with predominant PR $(n=13)$. For the PR subgroup, PVR led to not only improved LV circumferential strain, 
Table 4 Interobserver and intraobserver variability for global strain and synchrony parameters

\begin{tabular}{|c|c|c|c|c|}
\hline \multirow[t]{2}{*}{ Parameter } & \multicolumn{2}{|l|}{ Intraobserver $(n=10)$} & \multicolumn{2}{|l|}{ Interobserver $(n=10)$} \\
\hline & Mean difference \pm SD & ICC & Mean difference \pm SD & ICC \\
\hline \multicolumn{5}{|l|}{ Left ventricle } \\
\hline \multicolumn{5}{|l|}{ Circumferential - mid } \\
\hline Global strain (\%) & $0.25 \pm 1.3$ & 0.96 & $0.30 \pm 1.9$ & 0.89 \\
\hline Maximum difference T2P (ms) & $12.5 \pm 27.8$ & 0.86 & $1.0 \pm 38.5$ & 0.82 \\
\hline Cross-correlation delay (ms) & $2.8 \pm 21.9$ & 0.83 & $14.6 \pm 31.4$ & 0.62 \\
\hline \multicolumn{5}{|l|}{ Longitudinal } \\
\hline Global strain (\%) & $0.33 \pm 2.1$ & 0.93 & $1.6 \pm 3.7$ & 0.75 \\
\hline Maximum difference T2P (ms) & $102.3 \pm 145$ & 0.80 & $40.0 \pm 154$ & 0.67 \\
\hline Cross-correlation delay (ms) & $25.0 \pm 80.7$ & 0.73 & $45.8 \pm 107$ & 0.65 \\
\hline \multicolumn{5}{|l|}{ Right ventricle } \\
\hline \multicolumn{5}{|l|}{ Circumferential - mid } \\
\hline Global strain (\%) & $0.40 \pm 1.6$ & 0.95 & $0.31 \pm 2.0$ & 0.88 \\
\hline Maximum difference T2P (ms) & $64.0 \pm 126$ & 0.82 & $90.6 \pm 107.9$ & 0.61 \\
\hline Cross-correlation delay (ms) & $104 \pm 233$ & 0.66 & $93.0 \pm 230$ & 0.59 \\
\hline \multicolumn{5}{|l|}{ Longitudinal } \\
\hline Global strain (\%) & $1.0 \pm 2.8$ & 0.85 & $2.6 \pm 2.2$ & 0.76 \\
\hline Maximum difference T2P (ms) & $146 \pm 133$ & 0.76 & $157 \pm 158$ & 0.66 \\
\hline Cross-correlation delay (ms) & $22.4 \pm 85.9$ & 0.64 & $42.1 \pm 118$ & 0.53 \\
\hline
\end{tabular}

ICC intraclass correlation coefficient, $T 2 P$ time-to-peak, SD standard deviation

as it did in our patients with surgical PVR, but also improved longitudinal strain. This difference in longitudinal strain response may be related to the fact that even in the PR subgroup, there was some degree of pulmonary stenosis, more than in the current study population. There was no change in RV circumferential or longitudinal strain with transcatheter PVR in the PR subgroup, in agreement with our results for surgical PVR.

Prior studies assessing the impact of PVR on ventricular synchrony are also scarce. Lurz et al. examined 20 patients with RV-to-pulmonary artery conduit obstruction, most with TOF, before and after transcatheter PVR [14]. Based on tissue Doppler echocardiography from an apical 4-chamber view, the time-to-peak strain for the RV free wall decreased and for the LV free wall was unchanged, leading to significantly less interventricular mechanical delay following PVR. Again, the presence of RV outflow tract obstruction confounds the comparison to our results. However, similar to our study, the QRS duration was unchanged highlighting the importance of assessing both mechanical and electrical measures of ventricular synchrony.

\section{Clinical implications}

The clinical implications of improved LV circumferential strain and synchrony following PVR are uncertain but there are some data to suggest that this may be beneficial. Impaired LV systolic function in repaired TOF patients is fairly common [33, 34]. Both reduced LV global function as well as deformation indices have been shown to be important independent predictors of clinical deterioration, arrhythmia, and death in adults after TOF repair [3, 4, 24, 29, 34-36]. Moreover, our group has shown that LV dyssynchrony is associated with ventricular tachycardia and death in repaired TOF patients [12]. It is thus possible that the improvements in these parameters seen with PVR may lead to a better prognosis, and further study is warranted. For the RV in TOF, global systolic dysfunction is common and associated with adverse outcomes [4, 24]. Abnormalities in RV deformational indices are also prevalent $[10,37,38]$. Our data showed no improvement in any of these parameters with PVR. This finding suggests that strategies other than PVR may need to be pursued to achieve meaningful changes in outcomes related to impaired RV function. To this end, our data supports efforts to maintain pulmonary valve function and optimize myocardial preservation at the time of initial repair as later PVR cannot be relied on to restore function. Similarly, antifibrotic pharmacologic therapy should be explored as studies have implicated fibrosis in the pathophysiology of myocardial dysfunction [39-41]. The lack of improvement in RV functional indices may be because restoration of normal loading conditions occurred after the myocardium had sustained irreversible damage. Accordingly, future studies with larger numbers should assess whether earlier PVR leads to a better RV response. Lastly, our data 
showed that PVR did not lead to a convincing improvement in interventricular synchrony. Thus, for repaired TOF patients in whom interventricular dyssynchrony is thought to be playing a clinically important role, cardiac resynchronization therapy [42] and not simply PVR, may be needed. Future studies which focus on patients with marked interventricular dyssynchrony who undergo PVR are needed to definitely address this proposition.

\section{Limitations}

Several limitations of this study are worth noting. Assessments were performed at a mean of 7 months following PVR. Available data suggests that RV volumes and, thus, EF remain stable between 1 and 5 years after PVR [43]. Nevertheless, we cannot exclude the possibility that changes in strain and synchrony might develop with longer follow-up even in the absence of prosthetic valve dysfunction. The distal RV outflow tract wall was not analyzed because in many patients it consisted of thin patch material from the initial TOF repair and was not amenable to feature tracking analysis. The temporal resolution of the cine CMR data was relatively low; consequently, small changes in synchrony parameters may have gone undetected. However, as the temporal resolution for each patient was similar on the pre- and post-PVR CMR studies, the synchrony changes which were identified are likely valid. The small sample size of the study may have limited the ability to detect small changes in strain and synchrony parameters. Similarly, the power of our study was not sufficient to assess the influence of age at initial repair and age at PVR on the impact of PVR. Finally, a comparison between the PVR alone versus the PVR with RV remodeling cohorts was not reported owing to the small numbers in each subgroup. We acknowledge that RV remodeling may have some impact on regional mechanics, but our study was not adequately powered to address this question.

\section{Conclusions}

In this cohort, surgical PVR in repaired TOF patients resulted in improved LV global strain and no change in RV global strain. LV synchrony parameters improved or were unchanged, RV synchrony effects varied by location, and interventricular synchrony was largely unchanged. Future studies should address longer-term changes in strain and synchrony following PVR and their relationship to clinical outcomes.

\footnotetext{
Abbreviations

CCD: cross-correlation delay; CMR: cardiovascular magnetic resonance; EDV: end-diastolic volume; EF: ejection fraction; ESV: end-systolic volume; ICC: intraclass correlation coefficient; LV: left ventricle/left ventricular; PR: pulmonary regurgitation; PVR: pulmonary valve replacement; RV: right ventricle/right ventricular; TOF: Tetralogy of Fallot
}

\section{Funding}

This study was supported in part by the Higgins Family Research Fund. There are no relationships with industry.

\section{Availability of data and materials}

Please contact author for data requests.

\section{Authors' contributions}

SB: Study design, data acquisition and interpretation, writing of initial and final drafts and final approval of the manuscript. DH: Development of feature tracking technique for use in the laboratory, training of personnel for data acquisition and interpretation, contribution to interobserver variability analysis, review and final approval of the manuscript. BK: Data collection and interpretation, final approval of manuscript. EM: Development of a software package using LabVIEW ${ }^{\circledast}$ for cross-correlation analysis which was used as a component of synchrony analysis in this study as well as final approval of manuscript. PN: Design of the original randomized controlled trial and review and final approval of manuscript. TG: Conception and design of the original randomized controlled trial, review and final approval of manuscript. AP: Responsible for all aspects of the study. All authors read and approved the final manuscript.

\section{Ethics approval and consent to participate}

The Boston Children's Hospital Committee on Clinical Investigation granted permission for this study and waived the requirement for informed consent.

\section{Competing interests}

All authors declare that they have no competing interests.

\section{Publisher's Note}

Springer Nature remains neutral with regard to jurisdictional claims in published maps and institutional affiliations.

\section{Author details}

${ }^{1}$ Department of Cardiology, Boston Children's Hospital, Boston, USA. ${ }^{2}$ Department of Pediatrics, Harvard Medical School, Boston, USA. ${ }^{3}$ Department of Cardiac Surgery, Boston Children's Hospital, Boston, USA.

${ }^{4}$ Department of Surgery, Boston Children's Hospital, Boston, USA.

Received: 7 November 2017 Accepted: 22 May 2018

Published online: 18 June 2018

\section{References}

1. Egbe AC, Mittnacht AJ, Nguyen K, Joashi U. Risk factors for morbidity in infants undergoing tetralogy of fallot repair. Ann Pediatr Cardiol. 2014;7(1): $13-8$.

2. Cuypers JA, Menting ME, Konings EE, Opic P, Utens EM, Helbing WA, et al. Unnatural history of tetralogy of Fallot: prospective follow-up of 40 years after surgical correction. Circ. 2014;130(22):1944-53.

3. Geva T, Sandweiss BM, Gauvreau K, Lock JE, Powell AJ. Factors associated with impaired clinical status in long-term survivors of tetralogy of Fallot repair evaluated by magnetic resonance imaging. J Am Coll Cardiol. 2004; 43(6):1068-74

4. Valente AM, Gauvreau K, Assenza GE, Babu-Narayan SV, Schreier J, Gatzoulis MA, et al. Contemporary predictors of death and sustained ventricular tachycardia in patients with repaired tetralogy of Fallot enrolled in the INDICATOR cohort. Heart. 2014;100(3):247-53.

5. Cheung EW, Wong WH, Cheung YF. Meta-analysis of pulmonary valve replacement after operative repair of tetralogy of fallot. Am J Cardiol. 2010; 106(4):552-7.

6. Ferraz Cavalcanti PE, Sa MP, Santos CA, Esmeraldo IM, de Escobar RR, de Menezes AM, et al. Pulmonary valve replacement after operative repair of tetralogy of Fallot: meta-analysis and meta-regression of 3,118 patients from 48 studies. J Am Coll Cardiol. 2013;62(23):2227-43.

7. Gengsakul A, Harris L, Bradley TJ, Webb GD, Williams WG, Siu SC, et al. The impact of pulmonary valve replacement after tetralogy of Fallot repair: a matched comparison. Eur J Cardio-thorac Surg. 2007:32(3):462-8.

8. Harrild DM, Berul Cl, Cecchin F, Geva T, Gauvreau K, Pigula F, et al. Pulmonary valve replacement in tetralogy of Fallot: impact on survival and ventricular tachycardia. Circ. 2009;119(3):445-51. 
9. Therrien J, Siu SC, Harris L, Dore A, Niwa K, Janousek J, et al. Impact of pulmonary valve replacement on arrhythmia propensity late after repair of tetralogy of Fallot. Circ. 2001;103(20):2489-94.

10. Dragulescu A, Friedberg MK, Grosse-Wortmann L, Redington A, Mertens L. Effect of chronic right ventricular volume overload on ventricular interaction in patients after tetralogy of Fallot repair. J Am Soc Echocardiogr. 2014;27(8): 896-902.

11. Jing L, Haggerty CM, Suever JD, Alhadad S, Prakash A, Cecchin F, et al. Patients with repaired tetralogy of Fallot suffer from intra- and interventricular cardiac dyssynchrony: a cardiac magnetic resonance study. Eur Heart J Cardiovasc Imaging. 2014;15(12):1333-43.

12. Ortega M, Triedman JK, Geva T, Harrild DM. Relation of left ventricular dyssynchrony measured by cardiac magnetic resonance tissue tracking in repaired tetralogy of fallot to ventricular tachycardia and death. Am J Cardiol. 2011;107(10):1535-40.

13. Kempny A, Diller GP, Orwat S, Kaleschke G, Kerckhoff G, Bunck A, et al. Right ventricular-left ventricular interaction in adults with tetralogy of Fallot: a combined cardiac magnetic resonance and echocardiographic speckle tracking study. Int J Cardiol. 2012;154(3):259-64.

14. Lurz P, Puranik R, Nordmeyer J, Muthurangu V, Hansen MS, Schievano S, et al. Improvement in left ventricular filling properties after relief of right ventricle to pulmonary artery conduit obstruction: contribution of septal motion and interventricular mechanical delay. Eur Heart J. 2009;30(18):2266-74.

15. Tanaka H, Nesser HJ, Buck T, Oyenuga O, Janosi RA, Winter S, et al. Dyssynchrony by speckle-tracking echocardiography and response to cardiac resynchronization therapy: results of the speckle tracking and resynchronization (STAR) study. Eur Heart J. 2010;31(14):1690-700.

16. Taylor RJ, Umar F, Panting JR, Stegemann B, Leyva F. Left ventricular lead position, mechanical activation, and myocardial scar in relation to left ventricular reverse remodeling and clinical outcomes after cardiac resynchronization therapy: a feature-tracking and contrast-enhanced cardiovascular magnetic resonance study. Heart rhythm. 2016;13(2):481-9.

17. Harrild DM, Marcus E, Hasan B, Alexander ME, Powell AJ, Geva T, et al. Impact of transcatheter pulmonary valve replacement on biventricular strain and synchrony assessed by cardiac magnetic resonance feature tracking. Circ Cardiovasc Interv. 2013;6(6):680-7.

18. Hor KN, Gottliebson WM, Carson C, Wash E, Cnota J, Fleck R, et al. Comparison of magnetic resonance feature tracking for strain calculation with harmonic phase imaging analysis. JACC Cardiovasc Imaging. 2010;3(2): 144-51.

19. Liu B, Dardeer AM, Moody WE, Edwards NC, Hudsmith LE, Steeds RP. Normal values for myocardial deformation within the right heart measured by feature-tracking cardiovascular magnetic resonance imaging. Int J Cardiol. 2018;252:220-3.

20. Lu JC, Connelly JA, Zhao L, Agarwal PP, Dorfman AL. Strain measurement by cardiovascular magnetic resonance in pediatric cancer survivors: validation of feature tracking against harmonic phase imaging. Pediatr Radiol. 2014;44(9):1070-6.

21. Vo HD, Marwick TH, Negishi K. MRI-Derived Myocardial Strain Measures in Normal Subject. JACC Cardiovasc Imaging. 2018;11(2 Pt 1):196-205.

22. Geva T, Gauvreau K, Powell AJ, Cecchin F, Rhodes J, Geva J, et al. Randomized trial of pulmonary valve replacement with and without right ventricular remodeling surgery. Circ. 2010;122(11 Suppl):S201-8.

23. Samyn MM, Powell AJ, Garg R, Sena L, Geva T. Range of ventricular dimensions and function by steady-state free precession cine MRI in repaired tetralogy of Fallot: right ventricular outflow tract patch vs. conduit repair. Journal of magnetic resonance imaging : JMRI. 2007;26(4):934-40.

24. Moon TJ, Choueiter N, Geva T, Valente AM, Gauvreau K, Harrild DM. Relation of biventricular strain and dyssynchrony in repaired tetralogy of fallot measured by cardiac magnetic resonance to death and sustained ventricular tachycardia. Am J Cardiol. 2015;115(5):676-80.

25. Fornwalt BK, Arita T, Bhasin M, Voulgaris G, Merlino JD, Leon AR, et al. Cross correlation quantification of dyssynchrony: a new method for quantifying the synchrony of contraction and relaxation in the heart. J Am Soc Echocardiogr. 2007;20(12):1330-7.

26. Hor KN, Wansapura JP, Al-Khalidi HR, Gottliebson WM, Taylor MD, Czosek RJ, et al. Presence of mechanical dyssynchrony in Duchenne muscular dystrophy. J Cardiovasc Magn Reson. 2011;13:12.

27. Delfino JG, Fornwalt BK, Eisner RL, Leon AR, Oshinski JN. Cross-correlation delay to quantify myocardial dyssynchrony from phase contrast magnetic resonance (PCMR) velocity data. J Magn Reson Imaging : JMRI. 2008;28(5): 1086-91.

28. Geva T. Repaired tetralogy of Fallot: the roles of cardiovascular magnetic resonance in evaluating pathophysiology and for pulmonary valve replacement decision support. J Cardiovasc Magn Reson. 2011;13:9.

29. Diller GP, Kempny A, Liodakis E, Alonso-Gonzalez R, Inuzuka R, Uebing A, et al. Left ventricular longitudinal function predicts life-threatening ventricular arrhythmia and death in adults with repaired tetralogy of fallot. Circ. 2012;125(20):2440-6.

30. Harrild DM, Han Y, Geva T, Zhou J, Marcus E, Powell AJ. Comparison of cardiac MRI tissue tracking and myocardial tagging for assessment of regional ventricular strain. Int J Cardiovasc Imaging. 2012;28(8):2009-18.

31. Moody WE, Taylor RJ, Edwards NC, Chue CD, Umar F, Taylor TJ, et al. Comparison of magnetic resonance feature tracking for systolic and diastolic strain and strain rate calculation with spatial modulation of magnetization imaging analysis. J Magnetic Reson Imaging : JMRI. 2015; 41(4):1000-12

32. Coats L, Khambadkone S, Derrick G, Hughes M, Jones R, Mist B, et al. Physiological consequences of percutaneous pulmonary valve implantation: the different behaviour of volume- and pressure-overloaded ventricles. Eur Heart J. 2007;28(15):1886-93.

33. Broberg CS, Aboulhosn J, Mongeon FP, Kay J, Valente AM, Khairy P, et al. Prevalence of left ventricular systolic dysfunction in adults with repaired tetralogy of fallot. Am J Cardiol. 2011;107(8):1215-20.

34. Ghai A, Silversides C, Harris L, Webb GD, Siu SC, Therrien J. Left ventricular dysfunction is a risk factor for sudden cardiac death in adults late after repair of tetralogy of Fallot. J Am Coll Cardiol. 2002;40(9):1675-80.

35. Knauth AL, Gauvreau K, Powell AJ, Landzberg MJ, Walsh EP, Lock JE, et al. Ventricular size and function assessed by cardiac MRI predict major adverse clinical outcomes late after tetralogy of Fallot repair. Heart. 2008;94(2):211-6.

36. Orwat S, Diller GP, Kempny A, Radke R, Peters B, Kuhne T, et al. Myocardial deformation parameters predict outcome in patients with repaired tetralogy of Fallot. Heart. 2016:102(3):209-15.

37. Friedberg MK, Fernandes FP, Roche SL, Slorach C, Grosse-Wortmann L, Manlhiot C, et al. Relation of right ventricular mechanics to exercise tolerance in children after tetralogy of Fallot repair. Am Heart J. 2013;165(4):551-7.

38. Menting ME, van den Bosch AE, McGhie JS, Eindhoven JA, Cuypers JA, Witsenburg $M$, et al. Assessment of ventricular function in adults with repaired tetralogy of Fallot using myocardial deformation imaging. Eur Heart J Cardiovasc Imaging. 2015;16(12):1347-57.

39. Broberg CS, Huang J, Hogberg I, McLarry J, Woods P, Burchill L, et al. Diffuse LV myocardial fibrosis and its clinical associations in adults with repaired tetralogy of Fallot. JACC Cardiovasc Imaging. 2016;9(1):86-7.

40. Chen CA, Dusenbery SM, Valente AM, Powell AJ, Geva T. Myocardial ECV fraction assessed by CMR is associated with type of hemodynamic load and arrhythmia in repaired tetralogy of Fallot. JACC Cardiovasc Imaging. 2016; 9(1):1-10.

41. Pradegan N, Vida VL, Geva T, Stellin G, White MT, Sanders SP, et al. Myocardial histopathology in late-repaired and unrepaired adults with tetralogy of Fallot. Cardiovasc Pathol. 2016;25(3):225-31.

42. Kubus $P$, Materna $O$, Tax P, Tomek V, Janousek J. Successful permanent resynchronization for failing right ventricle after repair of tetralogy of Fallot. Circ. 2014;130(22):e186-90.

43. Hallbergson A, Gauvreau K, Powell AJ, Geva T. Right ventricular remodeling after pulmonary valve replacement: early gains, late losses. Ann Thorac Surg. 2015;99(2):660-6.

\section{Ready to submit your research? Choose BMC and benefit from}

- fast, convenient online submission

- thorough peer review by experienced researchers in your field

- rapid publication on acceptance

- support for research data, including large and complex data types

- gold Open Access which fosters wider collaboration and increased citations

- maximum visibility for your research: over $100 \mathrm{M}$ website views per year

At BMC, research is always in progress.

Learn more biomedcentral.com/submissions 